Open Access

\title{
Endothelial bioreactor system ameliorates multiple organ dysfunction in septic rats
}

\author{
Shuai $\mathrm{Ma}^{1,3 \dagger}$, Yuli Lin ${ }^{2 \dagger}$, Bo Deng ${ }^{1,3}$, Yin Zheng ${ }^{1,3}$, Chuanming Hao ${ }^{3}$, Rui He ${ }^{2^{*}}$ and Feng Ding ${ }^{1 *}$
}

\author{
* Correspondence: \\ ruihe@fudan.edu.cn; dingfeng@sjtu. \\ edu.cn \\ ${ }^{\dagger}$ Equal contributors \\ ${ }^{2}$ Department of Immunology, \\ Shanghai Medical College, Fudan \\ University, Shanghai 200032, China \\ 'Division of Nephrology \& Unit of \\ Critical Nephrology, Shanghai Ninth \\ People's Hospital, School of \\ Medicine, Shanghai Jiaotong \\ University, 639 Zhizaoju Road, \\ Shanghai 200011, China \\ Full list of author information is \\ available at the end of the article
}

\begin{abstract}
Background: The endothelium is a potentially valuable target for sepsis therapy. We have previously studied an extracorporeal endothelial cell therapy system, called the endothelial bioreactor (EBR), which prolonged the survival time of endotoxemia sepsis in swine. To further study of the therapeutic effects and possible mechanisms, we established a miniature EBR system for septic rats induced by cecal ligation and puncture (CLP).

Methods: In the miniature EBR system, the extracorporeal circulation first passed through a mini-hemofilter, and the ultrafiltrate (UF) was separated, then the UF passed through an EBR (a 1-mL cartridge containing approximately $2 \times 10^{6}$ endothelial cells grown on microcarriers) and interact with endothelial cells. Eighteen hours after $C L P$, the rats were treated for $4 \mathrm{~h}$ with this extracorporeal system containing either endothelial cells (EBR group) or no cells (sham EBR group). Physiologic and biochemical parameters, cytokines, endothelial functions, and 7-day survival time were monitored. In vitro, the pulmonary endothelial cells of the septic rats were treated with the EBR system and the resulting changes in their functions were monitored.

Results: The EBR system ameliorated CLP-induced sepsis compared with the sham EBR system. After CLP, the 7-day survival rate of sham-treated rats was only $25.0 \%$, while in the EBR-treated group, it increased to $57.1 \%(p=0.04)$. The EBR system protected the liver and renal function and ameliorated the kidney and lung injury. Meanwhile, this therapy reduced pulmonary vascular leakage and alleviated the infiltration of inflammatory cells in the lungs, especially neutrophils. Furthermore, after the EBR treatment both in vivo and in vitro, the expression of intercellular adhesion molecule-1 and the secretion of CXCL1 and CXCL2 of pulmonary endothelium decreased, which helped to alleviate the adhesion and chemotaxis of neutrophils. In addition, the EBR system decreased CD11b expression and intracellular free calcium level of peripheral blood neutrophils, modulated the activation of these neutrophils.

Conclusions: The EBR system significantly ameliorated CLP-induced sepsis and improved survival and organ functions. Compared with the sham EBR system, this extracorporeal endothelial therapy may be involved in modulating the function of pulmonary endothelial cells, reducing the adhesion and chemotaxis of neutrophil, and modulating the activation of peripheral blood neutrophils.

Keywords: Sepsis, Multiple organ dysfunction, Endothelial cells, Bioreactor, Extracorporeal circulation, Neutrophils, Inflammation
\end{abstract}




\section{Background}

Sepsis is defined as life-threatening organ dysfunction caused by a dysregulated host response to infection [1] and is the most common cause of death in the intensive care unit [2]. Despite the progress in the development of antibiotics and in critical care therapy, sepsis is still associated with a high mortality rate [2].

Cell-based therapy has developed into a new therapeutic platform for the treatment of a vast array of clinical disorders, which may prove to be a more successful strategy by providing a dynamic, interactive, and individualized therapeutic approach [3]. In animal studies, stem/progenitor technologies, including exogenous infusion and endogenous recruitment, have shown significant promise as treatment strategies in critical care medicine [4-7]. In terms of safety, extracorporeal cell therapies may be a better choice, which not only replace the function of injury cells or modulate the pathophysiological processes but also provide an immunoprotective barrier. In swine models of sepsis, extracorporeal cell therapy with granulocytes [8] or renal proximal tubule cells [9] improved survival duration. Furthermore, in a phase II multicenter clinical trial, involving critically ill patients with acute renal failure, this extracorporeal renal-tubule cell therapy was proved to be efficacious and safe [10]. Nevertheless, the difficulties in large animal experiments keep the mechanism study elucidation insufficient.

General dysfunction of the endothelium is a key event in the pathogenesis of sepsis [11]. Once the endothelium becomes activated during the development of sepsis, it transforms into a procoagulant, antifibrinolytic, and proadhesive state [12]. The endothelial activation in sepsis is associated with changes in hemostatic balance, leukocyte trafficking, vascular permeability, inflammatory processes, and microcirculatory flow [13]. Thus, the endothelium plays a key role in mediating the sepsis phenotype [14] and is a potentially valuable target of sepsis therapy.

We previously studied an endothelial cell therapy system [15], called the endothelial bioreactor (EBR), involving a nonwoven fabric polytetrafluoroethylene (PTFE) hollow fiber cartridge containing endothelial cells in an extracorporeal circuit. Timely use of EBR therapy may improve cardiovascular performance and prolong the survival time of endotoxemia sepsis in swine [15]. Nevertheless, the difficulties in large animal experiments keep its elucidation insufficient. Therefore, we established a miniature EBR system for septic rats to study the therapeutic effects and possible mechanisms of this endothelial cell-based therapy.

\section{Methods}

\section{Cecal ligation and puncture}

Adult male Sprague-Dawley rats (450-550 g) were used for the studies. The experimental procedures were in accordance with the Animal Care and Use Committee of Shanghai Jiaotong University, Shanghai, China. All surgical procedures were carried out under general anesthesia induced by $4 \%$ chloral hydrate $(0.9 \mathrm{~mL} / 100 \mathrm{~g}$ intraperitoneally). The cecum was identified and ligated with a 3-0 silk tie at $25 \%$ length of the cecum. A double puncture of the cecal wall was performed with a 20 -gauge needle, and the cecum was gently squeezed to ensure that a small amount of feces was extruded onto the surface of the bowel. Lactated Ringer's solution $(2 \mathrm{~mL} / 100 \mathrm{~g})$ was given 
subcutaneously as fluid resuscitation [16, 17]. As sham cecal ligation and puncture (CLP) rats, the cecum was minimally handled without ligation and puncture.

\section{Culturing of endothelial cells on microcarriers}

Endothelial cells (human umbilical vein endothelial cells (HUVECs)) were a gift from Professor Lijun Jia (Department of Immunology, Shanghai Medical College, Fudan University, Shanghai, China).

For endothelial cell culture, protocols were adapted based on the recommendations for microcarrier culture using Cytodex-3 (GE Healthcare, Piscataway, USA). Cytodex-3 is a microporous microcarrier, made up of a dextran matrix with a collagen layer at the surface, for expansion of cells. For stirred microcarrier cultures, $100-\mathrm{mL}$ spinner flasks (Bellco Glass, Vineland, USA) were used, with a final medium volume of $80 \mathrm{~mL}$ and a stirring speed of $40 \mathrm{rpm}$. Required quantities of Cytodex-3 were weighted (final concentrations of $1.0 \mathrm{mg} / \mathrm{mL}$ ), hydrated, and sterilized by autoclaving as recommended by the manufacturers. Microcarriers were equilibrated in the culture medium for at least 30 min prior to the addition of the cells in order to maximize cell attachment. The cells were cultured in DMEM media containing $10 \%$ heat-inactivated fetal bovine serum (GIBCO, Gaithersburg, USA), $100 \mathrm{U} / \mathrm{mL}$ penicillin, $100 \mu \mathrm{g} / \mathrm{mL}$ streptomycin, and $2 \mathrm{mM}$ glutamine. Incubation was conducted at $37{ }^{\circ} \mathrm{C}$ in a $5 \% \mathrm{CO}_{2}$, humidified atmosphere. The expansion of endothelial cells was performed as previously described [18].

\section{The experimental protocol of the EBR system}

Eighteen hours after CLP, the animals were re-anesthetized with chloral hydrate. The carotid artery and the femoral vein were isolated by dissection and cannulated with 0.97-mm polyethylene-50 tubing (Becton Dickinson, San Diego, USA) for implementation of extracorporeal circulation.

The EBR system consisted of a mini-hemofilter (polyether sulphone high-flux membrane, membrane surface area $0.02 \mathrm{~m}^{2}$, cutoff point $40 \mathrm{kD}$; PEONY, Shanghai, China), an EBR (a 1-mL cartridge containing approximately $2 \times 10^{6}$ endothelial cells grown on microcarriers), tubing lines, and mini-pumps (VWR, West Chester, USA).

Eighteen hours after CLP, these animals were randomly assigned to receive either EBR or sham treatment for $4 \mathrm{~h}$. The extracorporeal circulation was driven by a minipump from the carotid artery to the femoral vein at a blood flow rate of $0.8-1.0 \mathrm{~mL} /$ min [19]. In the EBR group, the extracorporeal circulation passed through a minihemofilter with the ultrafiltration rate at $0.25 \mathrm{~mL} / \mathrm{min}$, then the ultrafiltrate (UF) passed through an EBR (Fig. 1a, b). In the sham EBR group, the extracorporeal circulation was set up with the same hemofilter and bioreactor cartridge but without any cells. In the control group, all rats were without the CLP procedure or the extracorporeal circulation. A volume of $62.5 \mathrm{U} / \mathrm{mL}$ of heparin was used to prevent coagulation in this circuit. After $4 \mathrm{~h}$, the treatment was stopped and the rats were observed for recovery, returned to the animal facility, and given access to food and water. Survival time was assessed up to 7 days. Another group that underwent the shame CLP procedure without extracorporeal circulation was used as the control. Before and after the extracorporeal circulation, microcarriers were collected and stained with 4',6-diamidino-2phenylindole (DAPI; Becton Dickinson, San Diego, USA) and observed under a 

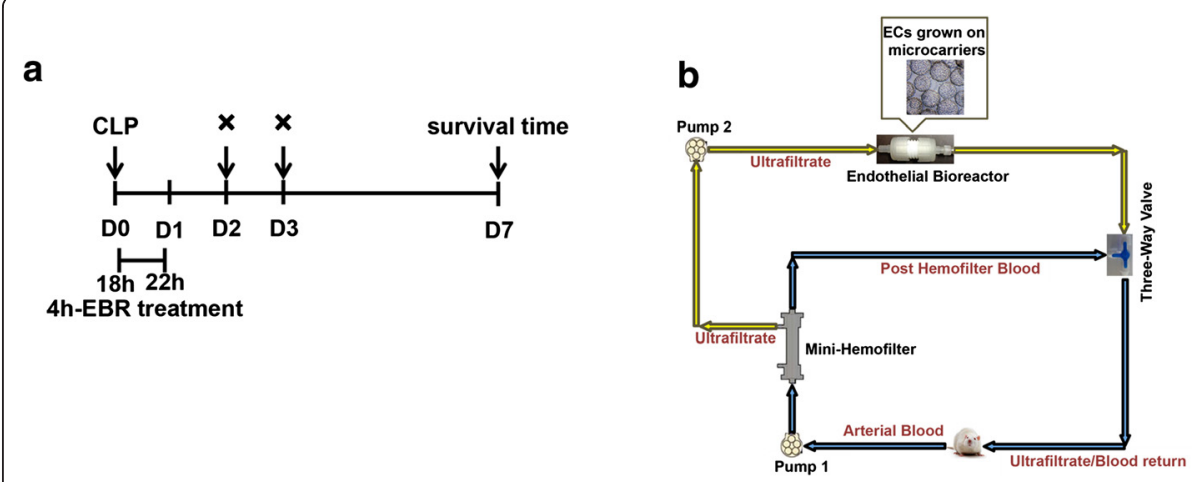

C
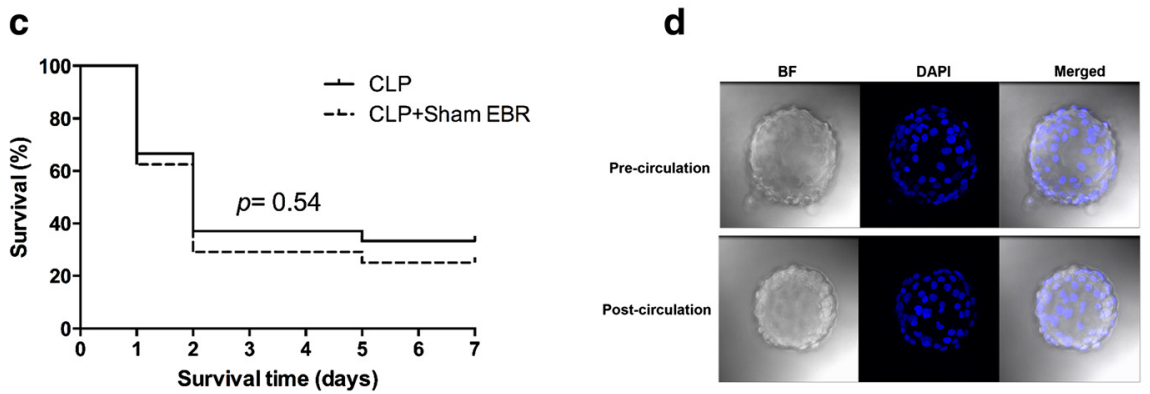

Fig. 1 Construction of the endothelial bioreactor (EBR) system for septic rats. a The protocol for the EBR treatment. b A schematic drawing of the EBR system. c Survival was plotted during a 7-day period $(n=22-$ 25 per group). $\mathbf{d}$ Endothelial cells grown on microcarriers were collected before and after the extracorporeal circulation and were stained with DAPI (blue) at $\times 400 \times Z 1.5$ magnification. CLP cecal ligation and puncture, EBR endothelial bioreactor, ECs endothelial cells, DAPI 4',6-diamidino-2-phenylindole, BF bright field

confocal laser scanning microscope (Leica Microsystems, Heidelberg, Germany) at a magnification of $\times 400 \times \mathrm{Z} 1.5$.

\section{Analysis of bronchoalveolar lavage fluid}

Bronchoalveolar lavage fluid (BALF) was obtained by lavaging the left lung once with $2 \mathrm{~mL}$ PBS and three times with $4 \mathrm{~mL}$ PBS. Total cells were counted under optical microscopes. Total protein in the first BALF was measured by bicinchoninic acid kit. TNF- $\alpha$, IL-1 $\beta$, and IL- 6 levels in the first BALF were measured by an enzyme-linked immunosorbent assay (ELISA) from eBioscience (San Diego, USA).

\section{Flow cytometric analysis}

Fluorochrome-labeled antibodies CD4, CD8, CD25, Foxp3, CD45, CD11b, and Granulocyte Marker (eBioscience, San Diego, USA) were used for surface and intracellular staining, according to the manufacturer's instructions. After staining, cells were analyzed by flow cytometer (Becton Dickinson, San Diego, USA).

\section{Quantitative real-time RT-PCR analysis}

Total RNA was extracted using Micro Scale RNAqueous Isolation kit, and then cDNA was synthesized with the High-Capacity cDNA Reverse Transcription kit (both from Applied Biosystems, Foster City, USA). Quantitative real-time polymerase chain reaction was performed with the SYBR green Gene Expression Assay (Applied Biosystems, 
Foster City, USA). The relative expressions of target genes were calculated using the $2^{\Delta} \mathrm{C}(\mathrm{t})$ method. The sequences of primers for polymerase chain reaction are as follows: intercellular adhesion molecule (ICAM)-1, 5' -CTCATCCTGCGCTGTCTGGT-3' (forward), 5'-CCGGAGCTGCCTGACCTCGG-3' (reverse); CXCL1, 5'-GCGTTTCATC GATGGTCGTT-3' (forward), 5'-CTTCTTCCCGCTCAACACCT-3' (reverse); and CXCL2, 5' -AACCATCAGGGTACAGGGGT-3' (forward), 5'-GGGCTTCAGGGTTG AGACAA-3' (reverse).

\section{Western blot analysis for ICAM-1}

Pulmonary endothelial cells or lung tissue were lysed with RIPA buffer. Proteins were separated by electrophoresis in a denaturing polyacrylamide gel and transferred to a PVDF membrane. After blocking with $5 \%$ milk-Tris-buffered saline and Tween 20 (TBST) and washing in TBST, membranes were then incubated in the appropriate primary antibodies (anti-ICAM-1; both from R\&D, Emeryville, USA) and anti-GAPDH (Cell Signal Technology, Beverly, USA) at $4{ }^{\circ} \mathrm{C}$ overnight. After washing, membranes were incubated with the appropriate HRP-conjugated secondary antibodies and analyzed by ECL development.

\section{Isolation of rat pulmonary endothelial cells}

The lungs were harvested at $18 \mathrm{~h}$ after CLP, finely minced, and digested in $10 \mathrm{ml}$ collagenase type II (2 mg/ml, Sigma-Aldrich, St. Louis, USA). The isolated cells were incubated with Anti-Rat CD31 Biotin (eBioscience, San Diego, USA), and endothelial cells bound with antibody were magnetically separated with Anti-Biotin Microbeads (Miltenyi Biotec, Bergisch Gladbach, Germany) as described previously [20, 21]. Pulmonary endothelial cells of healthy rats were also isolated as control.

\section{Simulation of EBR treatment in vitro}

Eighteen hours after CLP, the rats were cannulated, and the extracorporeal circulation passed through a mini-hemofilter with the ultrafiltration rate at $0.25 \mathrm{~mL} / \mathrm{min}$. The UF of the rats were harvested (sham-UF). The UF of healthy rats were also harvested as a control (control-UF). HUVECs were stimulated with the UF for $0,0.5,1,2$, or $4 \mathrm{~h}$. Then, the supernatant (EBR-UF) was collected and used to stimulate the isolated pulmonary endothelial cells. After $24 \mathrm{~h}$ of stimulation with EBR-UF, the cells were harvested for Western blot and the supernatant was used to analyze the concentrations of CXCL1 and CXCL2 using ELISA kits (R\&D, Emeryville, USA).

\section{Isolation of rat peripheral blood neutrophils and detection of intracellular free calcium} level

Rat neutrophils were isolated from the peripheral blood at $18 \mathrm{~h}$ after CLP by Percoll density gradient as previously described [22]. The purity of isolated neutrophils was checked by fluorochrome-labeled antibodies CD45 and Granulocyte Marker (eBioscience, San Diego, USA) using flow cytometer (Becton Dickinson, San Diego, USA). Neutrophils of healthy rats were also isolated as a control.

The neutrophils were incubated with Fluo-3 AM (Invitrogen) for $30 \mathrm{~min}$ in darkness at room temperature, and the resulting fluorescence as the indicator of $\mathrm{Ca}^{2+}$ was 
monitored using a flow cytometer (Becton Dickinson, San Diego, USA) at excitation wavelength $488 \mathrm{~nm}$ and the emission wavelength of $525 \mathrm{~nm}[23,24]$.

\section{Histopathologic analysis of the lung and kidney}

Lung sections were stained with hematoxylin and eosin. All lung fields at $\times 200$ magnification were examined for each sample. Assessment of histological lung injury was performed as follows: $1=$ normal; $2=$ focal ( $<50 \%$ lung section) interstitial congestion and inflammatory cell infiltration; $3=$ diffuse ( $>50 \%$ lung section) interstitial congestion and inflammatory cell infiltration; $4=$ focal ( $<50 \%$ lung section) consolidation and inflammatory cell infiltration; $5=$ diffuse ( $>50 \%$ lung section) consolidation and inflammatory cell infiltration [25].

Kidney sections were stained with periodic acid and Schiff's reagent. All kidney fields at $\times 400$ magnification were examined for each sample. Damaged renal tubules were identified by diffuse tubular dilatation, intraluminal casts and/or tubular cell blebbing, vacuolization, and detachment, in cortex and outer medulla, as follows: $0=$ none; $1=$ $<10 \% ; 2=11-25 \% ; 3=26-45 \% ; 4=46-75 \% ; 5=>76 \%[26,27]$.

\section{Measurements of the liver and renal functions}

Serum alanine aminotransferase (ALT), aspartate aminotransferase (AST), creatinine $(\mathrm{Cr})$, and blood urea nitrogen (BUN) were measured with an automated chemical analyzer (Vitros-950, Johnson \& Johnson, New Brunswick, USA).

\section{Statistical analysis}

All numerical data are expressed as mean \pm SEM. The Student's $t$ tests were used for comparisons between two groups. Multiple-group comparisons were performed by one-way ANOVA followed by a post hoc Tukey's test to compare each group. The survival analysis was performed by the Kaplan-Meier method and log-rank test. All statistical analyses were performed with GraphPad Prism software, and a two-sided $p<0.05$ was considered significant.

\section{Results}

\section{Construction of the EBR system for CLP-induced sepsis in rats}

In order to estimate the potential harmful effect of this extracorporeal circulation on survival time, the rats were divided into two groups: the CLP group (CLP, without extracorporeal circulation) and the sham EBR group (CLP, EBR system without any cells); then, survival was analyzed until 7 days after CLP. We found that survival duration was not statistically different between the sham EBR and control animals (25.0 vs. $33.3 \%, p=0.54$; Fig. 1c), which meant this circulation system was safe for the septic rats. The EBR was a 1-mL cartridge, whereas we need approximately $2 \times 10^{6}$ endothelial cells in it. Thus, we cultured endothelial cells on microcarriers to increase the surfacearea-to-volume ratio. During the culturing of endothelial cells on microcarriers, we analyzed the concentrations of glucose, lactate, and nitric oxide (NO) in different time points for analyzing the metabolic activity. We found that endothelial cells could grow adhering to the surface of microcarriers after seeding; lactate and NO levels in the culture media rose continuously, and the glucose concentration decreased with time, 
indicating metabolic activity residing in the microcarrier culture (data not shown). For testing whether the EBR system treatment will affect the adhesion of endothelial cells on the microcarrier, we collected microcarriers and stained them with DAPI before and after the extracorporeal circulation. We observed that there were a large number of endothelial cells growing on the microcarriers before the circulation. Similar observations after the extracorporeal circuit revealed no obvious decrease in the density of the cells (Fig. 1d). These data showed that we successfully constructed an EBR system for rats with CLP-induced sepsis.

\section{The EBR system ameliorated CLP-induced sepsis}

We next investigated whether this EBR system can ameliorate sepsis. First, we analyzed survival time after CLP. We found that the EBR system can improve the survival of rats with CLP-induced sepsis. Data showed that the 7-day survival rate of sham-treated rats (sham EBR group) was only $25.0 \%$, while in the EBR-treated group (EBR group), it increased to $57.14 \%$ ( $p=0.04$, sham EBR vs. EBR; Fig. 2a). Sepsis may result in multiple organ dysfunction, thus, we assessed the liver function, renal function, and histological injury of the kidney and lung. We monitored the liver function using serum ALT and AST and used serum $\mathrm{Cr}$ and BUN to monitor renal function. At $48 \mathrm{~h}$ after CLP, the levels of

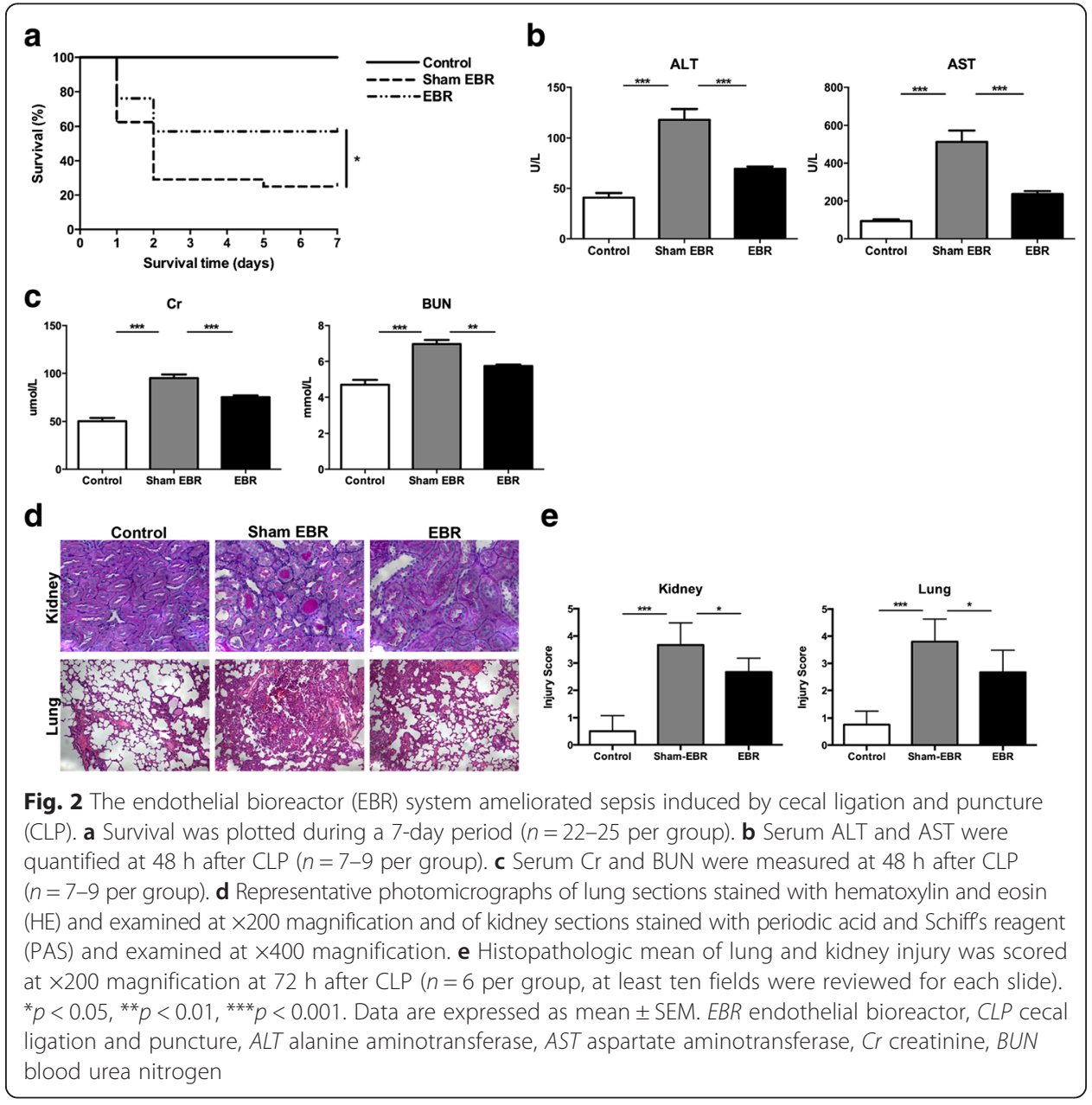


ALT and AST were elevated in the sham-EBR group compared with rats without induced sepsis or circulation (control group) ( $p<0.001$, sham EBR vs. control; Fig. 2b), while these metrics decreased significantly in the EBR-treated rats $(p<0.001$, sham EBR vs. EBR; Fig. 2b). Similarly, after the EBR treatment, the levels of serum $\mathrm{Cr}$ and BUN also decreased ( $p<0.01$, sham EBR vs. EBR; Fig. 2c). The degree of damaged renal tubules of the kidney and the pathology of the lung that includes interstitial congestion and inflammatory cell infiltration in sham-EBR group showed much severer injuries than the control group (Fig. 2d). However, the injuries were ameliorated after the EBR treatment (Fig. 2d). Furthermore, the injury scores of the kidney $(p<0.05$, sham EBR vs. EBR; Fig. $2 \mathrm{e})$ and lung ( $p<0.05$, sham EBR vs. EBR; Fig. 2e) confirmed the protective effects of the EBR system. These data suggested that the EBR system could prolong the survival time and reduce organ injuries of CLP-induced sepsis compared with the sham EBR system.

\section{The EBR system alleviated inflammation in the lungs}

The lung is one of the first organs to be affected in sepsis; cellular infiltration, along with the release of proinflammatory mediators, leads to the development of lung injury [5]. The concentration of total protein in BALF was used to assess pulmonary vascular leakage. At $48 \mathrm{~h}$ after CLP, the total protein levels in BALF were elevated in the shamEBR group compared with rats without induced sepsis or circulation (control group) $(p<0.001$, sham EBR vs. control; Fig. 3a), while the total protein decreased significantly

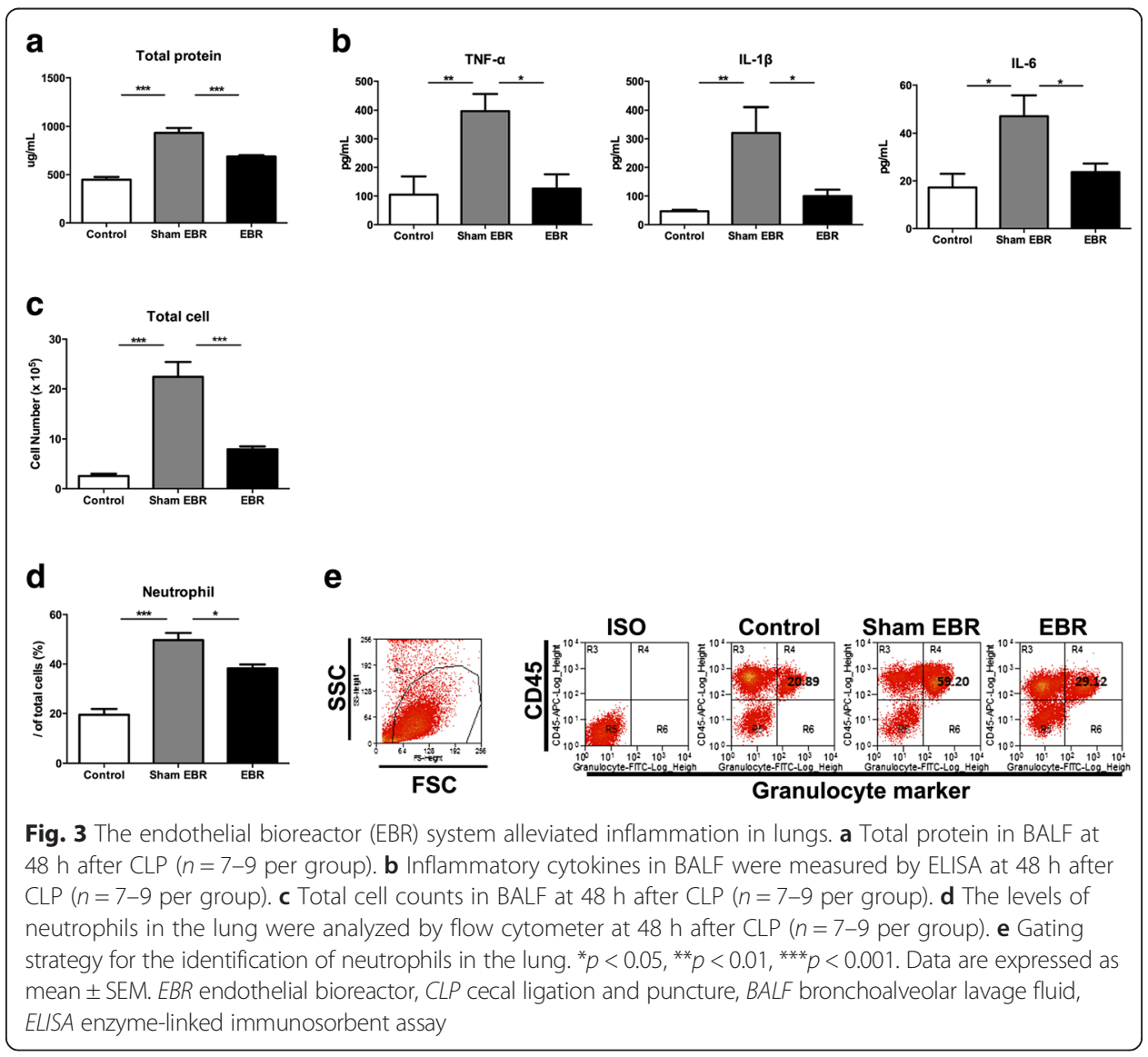


in the EBR-treated rats $(p<0.001$, sham EBR vs. EBR; Fig. 3a). As cytokine storm in the lungs may lead to lung injury [28], to further evaluate pulmonary inflammation in the septic rats, levels of inflammatory-associated cytokines including TNF- $\alpha$, IL-1 $\beta$, and IL6 were measured in the BALF samples. We found that the levels of these cytokines increased significantly in the sham-EBR group than the control group $(p<0.05$; Fig. $3 \mathrm{~b})$. EBR treatment could decrease the levels of TNF- $\alpha(p<0.05)$, IL-1 $\beta(p<0.05)$, and IL-6 $(p<0.05)$ compared with the sham-EBR group at $48 \mathrm{~h}$ after CLP (Fig. 3b). Considering that the upregulated proinflammatory cytokines can result in recruitment and activation of inflammatory cells [29], we counted the total cells in BALF of the different groups. We found that the total BALF cell count in the sham-EBR group was much more than that in the control group $(p<0.001$; Fig. $3 c)$. However, septic rats with EBR treatment showed a significant decrease in the total BALF cell count at $48 \mathrm{~h}$ after the CLP procedure compared with the sham EBR group ( $p<0.001$; Fig. $3 c$ ). In sepsis, activated neutrophils transmigrate and infiltrate into the lungs, and this overwhelming migration correlates with the severity of lung injury [30]. Thus, we examined the infiltration of neutrophils in the lungs by flow cytometer. Granulocytes marker [31] and CD45 double-positive cells were defined neutrophils in rats. We found that the infiltration of neutrophils in the lungs of EBR group was significantly reduced compared with sham-EBR group (38.2 vs. $49.7 \%, p<0.05$; Fig. 3d, e) at $48 \mathrm{~h}$ after CLP. Taken together, these data suggested that the EBR system could reduce lung injury by alleviating inflammation which includes inflammation-associated cytokines, cell numbers in BALF, and the infiltration of neutrophils in the lungs.

\section{The EBR system modulated the function of pulmonary endothelial cells and peripheral blood neutrophils}

Recruitment of neutrophils into the lung is a key event in the early development of acute lung injury [32]. CXCL1 and CXCL2 are dominant chemokines for attracting neutrophils in inflammatory diseases [32-34]. Thus, we analyzed the mRNA expressions of CXCL1 and CXCL2 in the lungs and found that the mRNA expressions of these two chemokines in the sham-EBR group was upregulated than the control group (CXCL1, $p<0.001$; CXCL2, $p<0.01$; Fig. 4a). However, the EBR system could significantly reduce the mRNA expression of CXCL1 and CXCL2 in the lung tissue compared with the sham EBR system (CXCL1, $p<0.001$; CXCL2, $p<0.05$; sham EBR vs. EBR; Fig. 4a). Acute inflammation is characterized by both neutrophil emigration and increased vascular permeability [35]. Activation of the vascular endothelium results in the induction of adhesion molecules (e.g., intercellular adhesion molecule-1 and Eselectin) and chemokines (e.g., CXCL1 and CXCL2) that play a central role in the cascade of leukocyte tethering, slow rolling, firm adhesion, and transendothelial migration [36, 37]. ICAM-1, a marker of pro-adhesive state endothelial cells, is highly expressed on endothelial cells in sepsis [38]. We found that the protein level of ICAM-1 (Fig. 4b) and mRNA expression of ICAM-1 $(p<0.001$; Fig. 4 c) in the sham-EBR group was upregulated than the control group, which implying the endothelial cells in the lung, were at pro-adhesive state after CLP. Furthermore, the EBR system could significantly reduce both the protein level of ICAM-1 (Fig. 4b) and the mRNA expression of ICAM-1 ( $p<$ 0.001, sham EBR vs. EBR; Fig. 4c) in the lung tissue compared with the sham EBR 


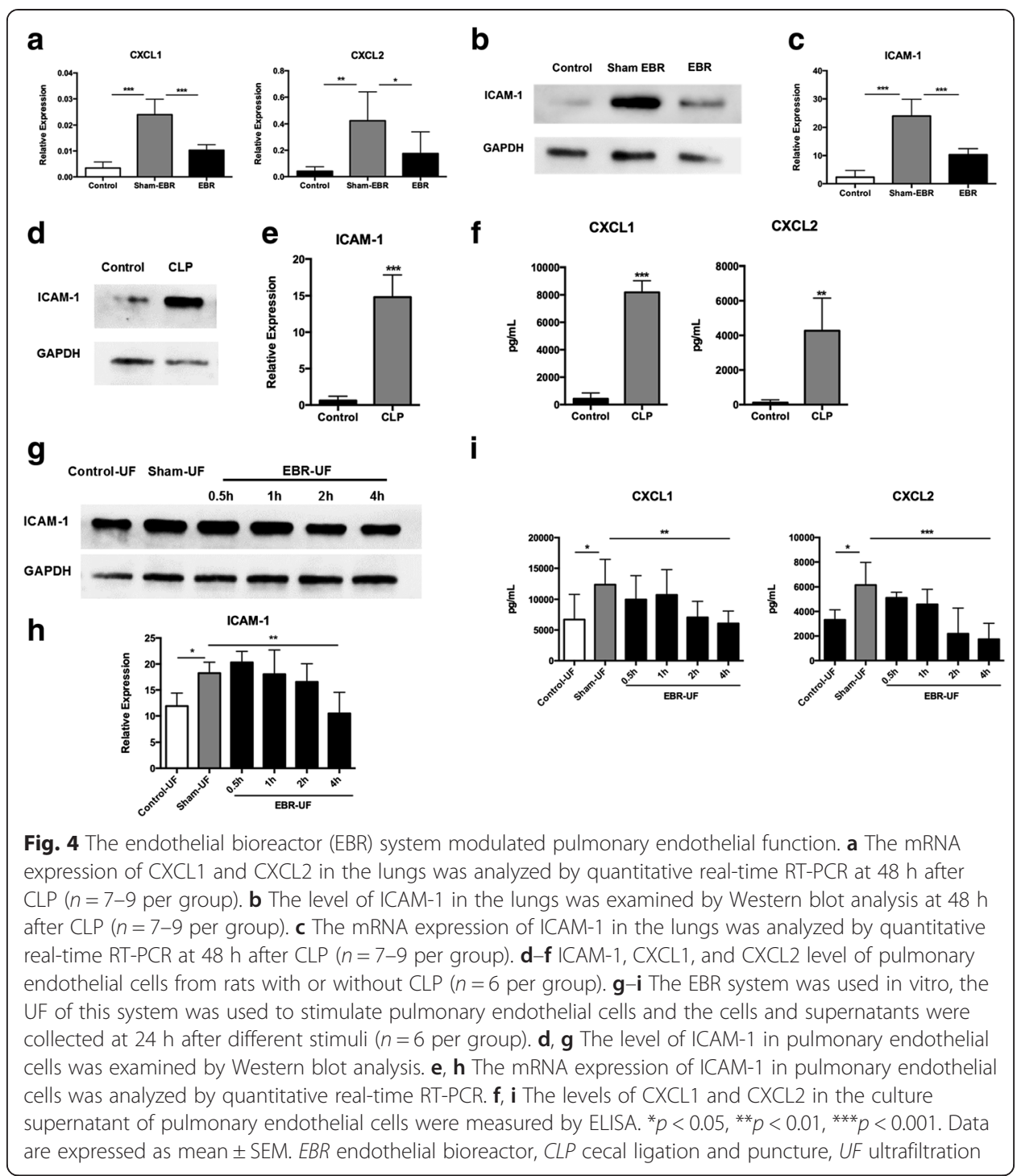

system, which suggested that the EBR system might ameliorate the pro-adhesive state. To further confirm that the EBR treatment may be involved in reducing the secretion of CXCL1 and CXCL2 and the expression of ICAM-1 of pulmonary endothelial cells, we isolated pulmonary endothelial cells from rats with or without CLP and mimicked the EBR system treatment in vitro by stimulating pulmonary endothelial cells with ultrafiltrate (UF) from control (control-UF), sham-EBR (sham-UF), and EBR group (EBRUF). We found that both of the protein level (Fig. 4d) and the mRNA expression of ICAM-1 ( $p<0.001$; Fig. 4e) were much higher in pulmonary endothelial cells of septic rats compared with the cells isolated from healthy rats. Stimulation with UF of the sham-EBR system increased the protein level and the mRNA expression of ICAM-1 ( $p$ $<0.05$, sham-UF vs. control-UF; Fig. $4 \mathrm{~g}, \mathrm{~h}$ ) in pulmonary endothelial cells of septic rats, whereas stimulation with UF of EBR system could reduce the level of ICAM-1 ( $p<$ 0.01, sham-UF vs. 4-h EBR-UF; Fig. 4g, h) in pulmonary endothelial cells of the septic rats. Meanwhile, we found that the levels of CXCL1 and CXCL2 in culture supernatant of pulmonary endothelial cells of septic rats increased significantly compared with the 
cells isolated from healthy rats (CXCL1, $p<0.001$; CXCL2, $p<0.01$; Fig. 4f). Stimulation with UF of sham-EBR system increased the levels of CXCL1 and CXCL2 $(p<0.05$, sham-UF vs. control-UF; Fig. 4i) in culture supernatant of pulmonary endothelial cells of septic rats, whereas stimulation with UF of EBR system could reduce the levels of these two chemokines (CXCL1, $p<0.01$; CXCL2, $p<0.001$; sham-UF vs. 4-h EBR-UF; Fig. 4i) in pulmonary endothelial cells of the septic rats. Collectively, these data suggested that the EBR system may be involved in modulating the function of pulmonary endothelial cells and reducing the adhesion and chemotaxis of neutrophils.

In addition, excessive activation of neutrophils is a major component of tissue damage and organ failure in sepsis [39]. CD11b and intracellular free calcium level are parameters commonly used for the assessment of neutrophil activation [40, 41]. Therefore, we isolated peripheral blood neutrophils from rats with CLP and stimulated with culture supernatant of HUVECs for $4 \mathrm{~h}$. The control groups were neutrophils without any stimulation. Then, the levels of CD11b and intracellular free calcium were analyzed by flow cytometer. The mean fluorescence intensity (MFI) of CD11b and the level of intracellular free calcium of peripheral blood neutrophils from CLP-rats increased significantly compared with neutrophils from healthy rats $(p<0.001, \mathrm{CLP}+$ and supernatant of HUVECs- vs. CLP- and supernatant of HUVECs-; Fig. 5a-d). However, these two parameters of neutrophils from septic rats decreased after stimulated with culture supernatant of HUVECs for $4 \mathrm{~h}$ compared with neutrophils with no stimulation (CD11b, $p<0.01 ; \mathrm{Ca}^{2+}, p<0.05 ; \mathrm{CLP}+$ and supernatant of HUVECs+ vs. CLP+ and supernatant of HUVECs-; Fig. 5a-d). These data show that the EBR system may be involved in modulating the activation of peripheral blood neutrophils.

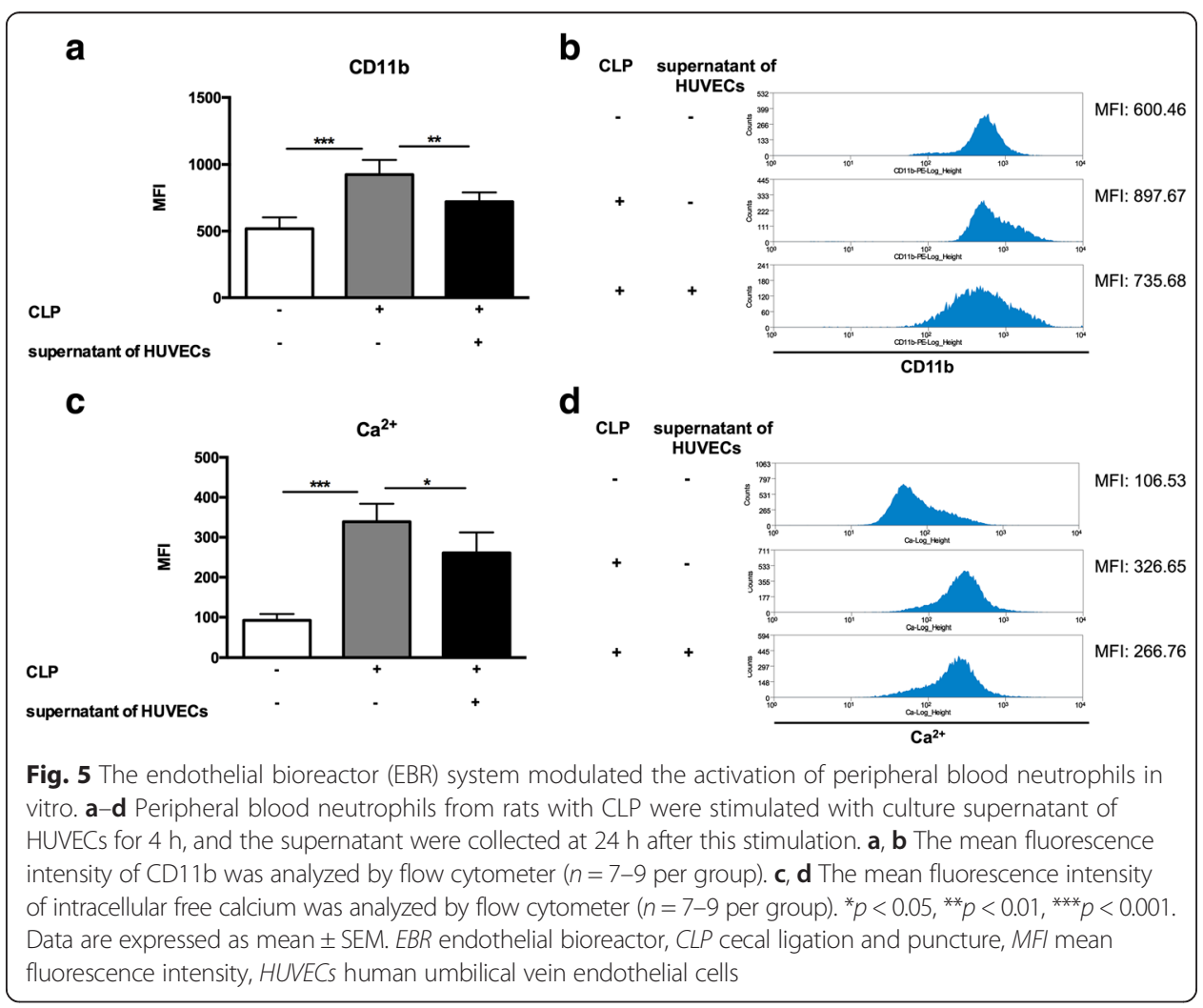




\section{Discussion}

A previous study by our laboratory has showed that timely EBR therapy improves cardiovascular performance and prolongs the survival time of endotoxemia sepsis in swine [15]. In the present study, we established a new EBR system for septic rats and demonstrated that the EBR system ameliorated CLP-induced sepsis and improved survival and organ functions. In animal models, the cytokine response is fully activated by $16-$ $18 \mathrm{~h}$ after CLP [42], and the ligated cecum begins to heal after $24 \mathrm{~h}$ [19, 43]. In clinical practice, patients often do not come to the hospital at the early stage of infection. In order to simulate clinical practice, we started the treatment at $18 \mathrm{~h}$ after CLP. The therapeutic use of extracorporeal bioreactors has a long tradition. Different cell-based extracorporeal organ support systems involving either hepatocytes or renal tubular cells have been used against acute liver failure [44-46] and acute renal failure associated with sepsis $[9,47]$. An extracorporeal bioartificial kidney consisting of a conventional hemofilter connected to a renal tubule assist device has shown its ability to successfully replace the filtration, transport, metabolic, and endocrine functions of the kidney in critically ill patients with acute renal failure in phase I/II clinical trials [10, 47]. Moreover, an extracorporeal treatment with donor granulocytes appears to be well tolerated and shows promising efficacy results in patients with septic shock, thus encouraging further studies [48].

The EBR system is an extracorporeal cell therapy with endothelial cells. We found that the EBR system alleviates inflammation in the lungs and reduces the infiltration of inflammatory cells, especially neutrophils. In sepsis, a number of different mechanisms may induce functional modulation of the endothelium, including increased expression of cell adhesion molecules and trafficking of leukocytes [49]. Endothelial cells are critical in maintaining a delicate balance between vasoconstriction and vasodilation, blood cell adherence and non-adherence, anticoagulation and procoagulation, and permeability and tightness [50]. These cells are not inert, rather they may adapt their function upon interaction with inflammatory mediators, and these changes are referred to as activation. They encompass a change from an anti- into a procoagulant surface; the expression of adhesion molecules; the production of inflammatory mediators, including chemoattractant agents; and the production of vasoactive compounds [11]. Two studies in cell-based treatments showed that transplanting endothelial cells to mice with ischemic acute kidney injury could change the function of renal endothelial cells and improve the renal function [51, 52]. The dysfunction or activation of endothelial cells plays a major role in the pathogenesis of sepsis $[11,14,50]$. Thus, endothelial cells represent an attractive therapeutic target in sepsis.

ICAM-1 is a cell-surface protein that is expressed at very low levels on pulmonary endothelium. The expression of ICAM-1 is upregulated after stimulation by inflammatory mediators such as cytokines and bacterial lipopolysaccharides during septic processes $[53,54]$. ICAM-1 mediates inflammatory responses via adhesion of leukocytes to activated endothelium and subsequent leukocyte transmigration through the pulmonary endothelial layer [55]. The increased expression of endothelial adhesion molecules either at the membrane level or in the plasma typifies different models of sepsis [12]. Moreover, there is a close relationship between plasma levels of adhesion molecules and outcomes of sepsis. In human sepsis, studies have shown that the higher the plasma levels of ICAM-1, the greater the number of organs damaged and the mortality 
$[13,56]$. The present study showed that the level of ICAM-1 in the lung tissue was increased after CLP, while the EBR treatment reduced its expression compared with the sham treatment. Furthermore, the level of pulmonary endothelial ICAM-1 significantly decreased after the EBR treatment in vitro. In other words, our findings indicate that this extracorporeal endothelial therapy may be involved in reducing the expression of ICAM-1 in the pulmonary endothelium, and this change should help to alleviate the adhesion of neutrophils. Moreover, in vivo and in vitro, we also found that this treatment decreased the secretion of CXCL1 and CXCL2 of septic pulmonary endothelial cells compared with the sham treatment. CXCL1 and CXCL2 are important neutrophil-attracting chemokines. Recent studies showed that CXCR2 (the receptor of CXCL1 and CXCL2) blockade results in impaired neutrophil recruitment in lipopolysaccharide-induced acute lung injury [57, 32]. Our data indicate that this EBR therapy may be involved in reducing the chemotaxis of neutrophils in the lung tissue via decreasing the secretion of CXCL1 and CXCL2 of pulmonary endothelial cells. Neutrophils are known to play an important role in inflammatory responses [58]. However, excessive activation of neutrophils is a direct cause of tissue damage and organ failure in sepsis $[39,58]$. Mac-1 (CD11b/CD18), the major subtype of integrins, is responsible for the firm adhesion of neutrophils to endothelium [40]. Once the neutrophils are activated, the shape of these cells changed and the amount of Mac-1 increased, resulting in enhanced adhesion to the endothelium and transmigration and infiltration of these neutrophils [40]. Another parameter commonly used for the assessment of neutrophil activation is intracellular free calcium level. $\mathrm{Ca}^{2+}$-dependent functions include activation of the membrane-associated superoxide-generating electron transporter, NADPH oxidase, and adhesion to the endothelium [41]. The present study showed that the EBR system decreased the CD11b expression and intracellular free calcium level of peripheral blood neutrophils compared with the sham treatment, which might modulate the activation of these neutrophils.

In the early phase of sepsis, the overwhelming inflammatory response is initiated after microbial infection [59], while the late phase is characterized by $\mathrm{T}$ cell hyporesponsiveness and defective antigen presentation [60,61]. This phase is considered a state of immunosuppression or immunoparalysis of the host [62]. Massive apoptosis of lymphocytes is one of the main drivers leading to immunoparalysis [63]. To assess the adaptive immune response, circulating and splenic levels of $\mathrm{CD} 4^{+}$helper $\mathrm{T}$ cells, $\mathrm{CD} 8^{+}$cytotoxic $\mathrm{T}$ cells, and $\mathrm{CD} 4^{+} \mathrm{CD} 25^{+} \mathrm{Foxp}^{+} \mathrm{T}$ regulatory cells were measured at $72 \mathrm{~h}$ after CLP. However, there was no significant difference between EBR-treated and sham-treated rats either in the peripheral circulation or in the spleen (Additional file 1: Figure S1). Thus, the 4-h EBR system treatment had no effect on the levels of T lymphocytes in the current rat model.

\section{Conclusions}

In summary, these data suggested that EBR system ameliorates CLP-induced sepsis and improves survival and organ functions compared with the sham EBR system. The EBR system alleviates inflammation in the lungs and reduces the infiltration of inflammatory cells and may be involved in modulating the function of pulmonary endothelial cells and reducing the adhesion and chemotaxis of neutrophils. In the same time, the EBR system might probably modulate the activation of peripheral blood neutrophils directly. 


\section{Additional file}

Additional file 1: Figure S1. The endothelial bioreactor (EBR) had no effect on the levels of T lymphocytes in septic rats. Circulating and splenic levels of $\mathrm{CD}^{+}$helper $\mathrm{T}$ cells, $\mathrm{CD} 8^{+}$cytotoxic $\mathrm{T}$ cells, and $\mathrm{CD} 4^{+} \mathrm{CD} 25^{+}$Foxp $^{+}{ }^{+}$ regulatory cells were analyzed by flow cytometer at $72 \mathrm{~h}$ after $\mathrm{CLP}(n=7-9$ per group). EBR $=$ endothelial bioreactor; $\mathrm{CLP}=$ cecal ligation and puncture. (TIF $307 \mathrm{~kb}$ )

\section{Acknowledgements}

We are grateful to Professor Lijun Jia (Department of Immunology, Shanghai Medical College, Fudan University, Shanghai, China) for providing HUVECs; Professor H. David Humes (Department of Internal Medicine, University of Michigan Medical School, Ann Arbor, Michigan, USA) for helping to construct the EBR system; and Doctor Zhiyong Peng and Professor John A. Kellum (The CRISMA Center, Department of Critical Care Medicine, University of Pittsburgh School of Medicine, Pittsburgh, Pennsylvania, USA) for supporting the extracorporeal circulation of rats.

\section{Funding}

This study was supported, in part, by grants from the National Natural Science Foundation of China (No: 81070609, 81270850, 81470990), the National Key Technology R\&D Program (No: 2011BAl10B08), the Shanghai Pudong New Area Program (No: PW2015D-4), and the Shanghai Jiaotong University Key Program (YG2014ZD06).

\section{Authors' contributions}

$F D, R H$, and $C H$ conceived and designed the research. $S M, B D, Y L$, and $Y Z$ performed the research. SM, BD, and FD analyzed the data. SM wrote the paper. All authors read and approved the final manuscript.

\section{Competing interests}

The authors declare that they have no competing interests.

\section{Author details}

'Division of Nephrology \& Unit of Critical Nephrology, Shanghai Ninth People's Hospital, School of Medicine, Shanghai Jiaotong University, 639 Zhizaoju Road, Shanghai 200011, China. ${ }^{2}$ Department of Immunology, Shanghai Medical College, Fudan University, Shanghai 200032, China. ${ }^{3}$ Division of Nephrology, Huashan Hospital, Fudan University, Shanghai 200040, China.

Received: 19 April 2016 Accepted: 13 July 2016

Published online: 22 July 2016

\section{References}

1. Singer M, Deutschman CS, Seymour CW et al (2016) The Third International Consensus Definitions for Sepsis and Septic shock (Sepsis-3). JAMA 315:801-810

2. Angus DC, Linde-Zwirble WT, Lidicker J et al (2001) Epidemiology of severe sepsis in the United States: analysis of incidence, outcome, and associated costs of care. Crit Care Med 29:1303-1310

3. Humes HD (2003) Cell Therapy: leveraging nature's therapeutic potential. J Am Soc Nephrol 14:2211-2213

4. Cribbs SK, Martin GS (2011) Stem cells in sepsis and acute lung injury. Am J Med Sci 341:325-332

5. Mei SH, Haitsma JJ, Dos Santos CC et al (2010) Mesenchymal stem cells reduce inflammation while enhancing bacterial clearance and improving survival in sepsis. Am J Respir Crit Care Med 182:1047-1057

6. Gupta N, Su X, Popov B et al (2007) Intrapulmonary delivery of bone marrow-derived mesenchymal stem cells improves survival and attenuates endotoxin-induced acute lung injury in mice. J Immunol 179:1855-1863

7. Kähler CM, Wechselberger J, Hilbe W et al (2007) Peripheral infusion of rat bone marrow derived endothelial progenitor cells leads to homing in acute lung injury. Respir Res 8:50

8. Sauer M, Altrichter J, Kreutzer $\mathrm{HJ}$ et al (2009) Extracorporeal cell therapy with granulocytes in a pig model of Gram-positive sepsis. Crit Care Med 37:606-613

9. Humes HD, Buffington DA, Lou L et al (2003) Cell therapy with a tissue-engineered kidney reduces the multipleorgan consequences of septic shock. Crit Care Med 31:2421-2428

10. Tumlin J, Wali R, Williams W et al (2008) Efficacy and safety of renal tubule cell therapy for acute renal failure. J Am Soc Nephrol 19:1034-1040

11. Hack CE, Zeerleder S (2001) The endothelium in sepsis: source of and a target for inflammation. Crit Care Med 29: S21-S27

12. Ait-Oufella H, Maury E, Lehoux S et al (2010) The endothelium: physiological functions and role in microcirculatory failure during severe sepsis. Intensive Care Med 36:1286-1298

13. Skibsted S, Jones AE, Puskarich MA et al (2013) Biomarkers of endothelial cell activation in early sepsis. Shock 39: $427-432$

14. Aird WC (2003) The role of the endothelium in severe sepsis and multiple organ dysfunction syndrome. Blood 101:3765-3777

15. Xie Q, Liu J, Gu Y et al (2010) Endothelial bioreactor ameliorates endotoxemia sepsis in swine. Blood Purif 29: 252-258

16. Brooks HF, Osabutey CK, Moss RF et al (2007) Caecal ligation and puncture in the rat mimics the pathophysiological changes in human sepsis and causes multi-organ dysfunction. Metab Brain Dis 22:353-373

17. Herzig DS, Driver BR, Fang G et al (2012) Regulation of lymphocyte trafficking by CXC chemokine receptor 3 during septic shock. Am J Respir Crit Care Med 185:291-300

18. Abranches E, Bekman E, Henrique D, Cabral JM (2007) Expansion of mouse embryonic stem cells on microcarriers. Biotechnol Bioeng 96:1211-1221 
19. Peng ZY, Wang HZ, Carter MJ et al (2012) Acute removal of common sepsis mediators does not explain the effects of extracorporeal blood purification in experimental sepsis. Kidney Int 81:363-369

20. Gandhirajan RK, Meng S, Chandramoorthy HC et al (2013) Blockade of NOX2 and STIM1 signaling limits lipopolysaccharide-induced vascular inflammation. J Clin Invest 123:887-902

21. King J, Hamil T, Creighton J et al (2004) Structural and functional characteristics of lung macro- and microvascular endothelial cell phenotypes. Microvasc Res 67:139-151

22. Sethi S, Singh MP, Dikshit M (1999) Nitric oxide-mediated augmentation of polymorphonuclear free radical generation after hypoxia-reoxygenation. Blood 93:333-340

23. Li SP, Xie WL, Cai HH et al (2012) Hydroxyl radical scavenging mechanism of human erythrocytes by quercetingermanium (IV) complex. Eur J Pharm Sci 47:28-34

24. Jin H, Yang P, Cai J et al (2012) Photothermal effects of folate-conjugated Au nanorods on HepG2 cells. Appl Microbiol Biotechnol 94:1199-1208

25. D'Alessio FR, Tsushima K, Aggarwal NR et al (2009) $C D 4^{+} \mathrm{CD} 25^{+} \mathrm{Foxp}^{+}$Tregs resolve experimental lung injury in mice and are present in humans with acute lung injury. J Clin Invest 119:2898-2913

26. Gandolfo MT, Jang HR, Bagnasco SM et al (2009) Foxp3 ${ }^{+}$regulatory T cells participate in repair of ischemic acute kidney injury. Kidney Int 76:717-729

27. Hu J, Zhang L, Wang $\mathrm{N}$ et al (2013) Mesenchymal stem cells attenuate ischemic acute kidney injury by inducing regulatory T cells through splenocyte interactions. Kidney Int 84:521-531

28. Bosmann M, Ward PA (2012) Role of C3, C5 and anaphylatoxin receptors in acute lung injury and in sepsis. Adv Exp Med Biol 946:147-159

29. Shubin NJ, Monaghan SF, Ayala A (2011) Anti-inflammatory mechanisms of sepsis. Contrib Microbiol 17: $108-124$

30. Hirano Y, Aziz M, Yang WL et al (2015) Neutralization of osteopontin attenuates neutrophil migration in sepsisinduced acute lung injury. Crit Care 19:53

31. Kanno E, Kawakami K, Ritsu M et al (2011) Wound healing in skin promoted by inoculation with Pseudomonas aeruginosa PAO1: the critical role of tumor necrosis factor-a secreted from infiltrating neutrophils. Wound Repair Regen 19:608-621

32. Reutershan J, Morris MA, Burcin TL et al (2006) Critical role of endothelial CXCR2 in LPS-induced neutrophil migration into the lung. J Clin Invest 116:695-702

33. Zhou H, Andonegui G, Wong CH et al (2009) Role of endothelial TLR4 for neutrophil recruitment into central nervous system microvessels in systemic inflammation. J Immunol 183:5244-5250

34. Wu F, Zhao Y, Jiao T et al (2015) CXCR2 is essential for cerebral endothelial activation and leukocyte recruitment during neuroinflammation. J Neuroinflammation 12:98

35. Yao L, Yago T, Shao B et al (2013) Elevated CXCL1 expression in gp130-deficient endothelial cells impairs neutrophil migration in mice. Blood 122:3832-3842

36. Pober JS, Cotran RS (1990) Cytokines and endothelial cell biology. Physiol Rev 70:427-451

37. Griffin GK, Newton G, Tarrio ML et al (2012) IL-17 and TNF-a sustain neutrophil recruitment during inflammation through synergistic effects on endothelial activation. J Immunol 188:6287-6299

38. Kayal S, Jaïs JP, Aguini N et al (1998) Elevated circulating E-selectin, intercellular adhesion molecule 1, and von Willebrand factor in patients with severe infection. Am J Respir Crit Care Med 157:776-784

39. Luo L, Zhang S, Wang Y et al (2014) Proinflammatory role of neutrophil extracellular traps in abdominal sepsis. Am J Physiol Lung Cell Mol Physiol 307:L586-L596

40. Shen YC, Chen CF, Sung YJ (1999) Tetrandrine ameliorates ischaemia-reperfusion injury of rat myocardium through inhibition of neutrophil priming and activation. Br J Pharmacol 128:1593-1601

41. Tintinger G, Steel HC, Anderson R (2005) Taming the neutrophil: calcium clearance and influx mechanisms as novel targets for pharmacological control. Clin Exp Immunol 141:191-200

42. Hubbard WJ, Choudhry M, Schwacha MG et al (2005) Cecal ligation and puncture. Shock 24(Suppl 1):52-57

43. Maier S, Traeger T, Entleutner M et al (2004) Cecal ligation and puncture versus colon ascendens stent peritonitis: two distinct animal models for polymicrobial sepsis. Shock 21:505-511

44. Allen JW, Hassanein T, Bhatia SN (2001) Advances in bioartificial liver devices. Hepatology 34:447-455

45. Strain AJ, Neuberger JM (2002) A bioartificial liver-state of the art. Science 295:1005-1009

46. Demetriou AA, Brown RS Jr, Busuttil RW et al (2004) Prospective, randomized, multicenter, controlled trial of a bioartificial liver in treating acute liver failure. Ann Surg 239:660-667

47. Humes HD, Weitzel WF, Bartlett RH et al (2004) Initial clinical results of the bioartificial kidney containing human cells in ICU patients with acute renal failure. Kidney Int 66:1578-1588

48. Altrichter J, Sauer M, Kaftan K et al (2011) Extracorporeal cell therapy of septic shock patients with donor granulocytes: a pilot study. Crit Care 15:R82

49. Vallet B, Wiel E (2001) Endothelial cell dysfunction and coagulation. Crit Care Med 29:S36-S41

50. Volk T, Kox WJ (2000) Endothelium function in sepsis. Inflamm Res 49:185-198

51. Brodsky SV, Yamamoto T, Tada T et al (2002) Endothelial dysfunction in ischemic acute renal failure: rescue by transplanted endothelial cells. Am J Physiol Renal Physiol 282:F1140-F1149

52. Herrler $\mathrm{T}$, Wang $\mathrm{H}$, Tischer A et al (2013) Decompression of inflammatory edema along with endothelial cell therapy expedites regeneration after renal ischemia-reperfusion injury. Cell Transplant 22:2091-2103

53. Osborn L (1990) Leukocyte adhesion to endothelium in inflammation. Cell 62:3-6

54. Tsokos M, Fehlauer F (2001) Post-mortem markers of sepsis: an immunohistochemical study using VLA-4 (CD49d/CD29) and ICAM-1 (CD54) for the detection of sepsis-induced lung injury. Int J Legal Med 114 291-294

55. Carlos TM, Harlan JM (1994) Leukocyte-endothelial adhesion molecules. Blood 84:2068-2101

56. Sessler CN, Windsor AC, Schwartz M et al (1995) Circulating ICAM-1 is increased in septic shock. Am J Respir Crit Care Med 151:1420-1427

57. Zarbock A, Allegretti M, Ley K (2008) Therapeutic inhibition of CXCR2 by reparixin attenuates acute lung injury in mice. Br J Pharmacol 155:357-364 
58. Souto FO, Alves-Filho JC, Turato WM et al (2011) Essential role of CCR2 in neutrophil tissue infiltration and multiple organ dysfunction in sepsis. Am J Respir Crit Care Med 183:234-242

59. de Jong HK, van der Poll T, Wiersinga WJ (2010) The systemic pro-inflammatory response in sepsis. J Innate Immun 2:422-430

60. Bone RC (1996) Sir Isaac Newton, sepsis, SIRS, and CARS. Crit Care Med 24:1125-1128

61. Zhu J, Wang J, Sheng Y et al (2012) Baicalin improves survival in a murine model of polymicrobial sepsis via suppressing inflammatory response and lymphocyte apoptosis. PLoS One 7:e35523

62. Rittisch D, Flierl MA, Ward PA (2008) Harmful molecular mechanisms in sepsis. Nat Rev Immunol 8:776-786

63. Hotchkiss RS, Osmon SB, Chang KC et al (2005) Accelerated lymphocyte death in sepsis occurs by both the death receptor and mitochondrial pathways. J Immunol 174:5110-5118

Submit your manuscript to a SpringerOpen ${ }^{\circ}$ journal and benefit from:

- Convenient online submission

- Rigorous peer review

- Immediate publication on acceptance

- Open access: articles freely available online

- High visibility within the field

- Retaining the copyright to your article

Submit your next manuscript at $>$ springeropen.com 